\title{
Spin-polarized ballistic transport in diluted magnetic semiconductor quantum wire systems
}

\author{
Kai Chang ${ }^{1,2, *}$ and F. M. Peeters ${ }^{1}$ \\ ${ }^{1}$ Department of Physics, University of Antwerp (UIA), B-2610 Antwerpen, Belgium \\ ${ }^{2}$ NLSM, Institute of Semiconductors, Chinese Academy of Sciences, P.O. Box 912, Beijing 100083, China
}

(Received 19 May 2003; published 21 November 2003)

\begin{abstract}
The energy dispersion of an electron in a double quantum wire with a diluted magnetic semiconductor barrier in between is calculated. An external magnetic field modifies significantly the energy dispersion of the electron which is different for the two spin states. The conductance exhibits many interesting peaks and dips which are directly related to the energy dispersions of the different electron spin states. These phenomena are attributed to the interwell coupling which can be tuned by the magnetic field due to the $s-d$ exchange interaction.
\end{abstract}

DOI: 10.1103/PhysRevB.68.205320

PACS number(s): 73.21.Fg, 75.50.Pp, 71.35.Ji, 78.20.Ls

Diluted magnetic semiconductors (DMS) have attracted much attention recently due to its spin-dependent transport properties and its potential applications in spintronic devices. ${ }^{1}$ Most previous investigations focus on the spinpolarized tunneling through diluted magnetic semiconductor junctions. ${ }^{2}$ Up to $90 \%$ polarization of the tunneling current was found. Tunneling occurred through a DMS junction in the presence of a magnetic field. The large polarization was due to the strong $s p-d$ exchange interaction between the local magnetic impurity and the electrons. This exchange interaction gives rise to a spin-dependent potential and lifts the degeneracy of the spin-up and spin-down electron states, consequently influencing the transport properties of the DMS.

The in-plane spin injection is extremely attractive for planar devices with submicron dimensions, where the mean free length for the momentum scattering can easily exceed the dimensions of the device. The current in narrow quantum wires is carried by a small number of transverse subbands. ${ }^{5}$ Most previous studies on the in-plane spin injection concentrated on planar hybrid structures of ferromagnetic metal and semiconductor. ${ }^{3,4}$ In this work we study the ballistic transport properties of electrons in DMS double quantum wire (DQW) structures in the presence of a magnetic field. We find that the energy dispersion of the electron can be tuned by an external magnetic field, which is quite different for the different spin orientations. The ground state of the spin-up electron shifts from zero in-plane momentum to a finite in-plane momentum when the in-plane magnetic field increases. But for the spin-down electron the ground state is still located at zero in-plane momentum. The conductance exhibits interesting structures which relate to the interwire coupling and the electron spin orientation in DMS DQW. Consequently the variation of the energy dispersion leads to changes in the conductance, i.e., spin-polarized peaks and dips are found with increasing Fermi energy and magnetic field.

As an example we consider a DQW consisting of $\mathrm{Zn}_{1-x} \mathrm{Cd}_{x} \mathrm{Se}$ wires separated by a DMS barrier $\mathrm{Zn}_{0.9} \mathrm{Mn}_{0.1} \mathrm{Se}$ and surrounded by $\mathrm{ZnSe}$. The structure is shown schematically in the inset of Fig. 3(a). The origin of the coordinate system is taken in the center of the DQW, i.e., at the center of the middle barrier, and we choose the $z$ axis perpendicular to the wire direction ( $y$ axis) and parallel to the magnetic field. The electron Hamiltonian in a DQW structure can be written as

$$
\begin{aligned}
H_{e} & =\frac{p_{x}^{2}}{2 m_{e}^{*}}+\frac{\left(p_{y}-e B x\right)^{2}}{2 m_{e}^{*}}+V_{e}(x)+J_{s-d} \mathbf{S} \cdot \mathbf{S}, \\
& =\frac{p_{x}^{2}}{2 m_{e}^{*}}+\frac{m_{e}^{*} \omega^{2}\left(x-x_{0}\right)^{2}}{2}+V_{e}(x)+V_{s-d}, \\
& =\frac{p_{x}^{2}}{2 m_{e}^{*}}+V_{e f f}\left(x, p_{y}\right),
\end{aligned}
$$

where $m_{e}^{*}$ is the effective mass of the electron in units of the free electron mass $m_{0} . V_{e}$ is the confining potential of the electron in the double quantum wire, i.e., $V_{e}\left(z_{e}\right)=V_{b}^{0}$ for $|z| \leqslant d / 2$ and $V_{e}^{0}$ for $|z| \geqslant W+d / 2 ; \quad V_{e}\left(z_{e}\right)=0$ otherwise, where $W$ is the width of the single well and $d$ is the thickness of the DMS layer. Within the molecular-field approximation, the exchange interaction [the fourth term in Eq. (1)] $V_{s-d}=J_{s-d}\left\langle S_{z}\left(z_{e}\right)\right\rangle s_{z}$ describes the $s-d$ exchange interaction between the electrons and the magnetic ions in the middle barrier region. Here $J_{s-d}=N_{0} \alpha x_{e f f}$ and $\left\langle S_{z}\right\rangle$ $=5 / 2 B_{J}\left[S g_{\mathrm{Mn}} \mu_{B} B / k_{B}\left(T+T_{0}\right)\right]$, where $S=5 / 2$ is the spin of the localized $3 d^{5}$ electrons of the $\mathrm{Mn}^{2+}$ ions. $B_{J}(x)$ is the Brillouin function, $N_{0}$ is the number of cations per unit volume, is the $x_{e f f}$ the phenomenological parameters (reduced effective concentration of $\mathrm{Mn}$ ) $T_{0}$ accounts for the reduced single-ion contribution due to the antiferromagnetic $\mathrm{Mn}-\mathrm{Mn}$ coupling, $k_{B}$ is the Boltzmann constant, $\mu_{B}$ is the Bohr magneton, $g_{\mathrm{Mn}}=2$ is the $g$ factor of the $\mathrm{Mn}^{2+}$ ions, and $s_{z}=$ $\pm 1 / 2$ is the electron spin. The parameters used in our calculations are $m_{e}^{*}=0.13 m_{0}, x_{e f f}=0.04, N_{0} \alpha=0.27 \mathrm{eV}, T_{0}$ $=1.5 \mathrm{~K}, V_{b}^{0}=6 \mathrm{meV}$, and $V_{e}^{0}=180 \mathrm{meV} \cdot{ }^{6-8}$

The envelope wave function is expanded by a series of sine functions defined in a hard-wall well with its width $D$ much larger than the cyclotron-orbit radius

$$
\phi(x)=\sqrt{\frac{2}{D}} \sum C_{n} \sin \left[\frac{n \pi}{D}(x+D / 2)\right]
$$


where $D \approx(2-3) L, L=2 W+d$. All elements of the Hamitonian can be calculated analytically. In our calculation we take typically $n=400$ [see Eq. (4)] which leads to an accuracy for the ground-state energy better than $0.01 \%$.

The electron conductance for ballistic transport is obtained from the Büttiker formulism ${ }^{9}$

$$
G=\left(2 e^{2} / h\right) N\left(E_{F}\right),
$$

where $N\left(E_{F}\right)$ is the number of occupied conduction channels along the positive direction of the $y$ axis. The group velocity of the electrons in the $n$th subband is proportional to the slope of the subband: $v_{n}=\partial E_{n}\left(k_{y}\right) / \hbar \partial k_{y}$. The polarization of the current is defined as $\alpha=\left(G_{\downarrow}-G_{\uparrow}\right) /\left(G_{\downarrow}+G_{\uparrow}\right)$. The Fermi energy can be obtained from by solving the following equation for a fixed density

$$
n_{e}=\frac{1}{2 \pi} \sum \int_{-k_{F}}^{k_{F}} f_{n}(k) d k
$$

where $f_{n}(k)=1 /\left[1+\exp \left(-\left(E-E_{F}\right) / k_{B} T\right)\right]$ is the FermiDirac distribution of electron in the $n$th band, and $n_{e}$ is the density of electron in DMS DQW.

In Figs. 1(a) and 1(b) we plot the energy dispersions of spin-up and spin-down electrons for two different magnetic fields as a function of the momentum along the $y$ axis. At small magnetic field, the energy dispersion of the electrons for different spin orientations is similar. With increase of magnetic field, spin-up and spin-down electrons exhibit very different energy dispersions for small in-plane momentum $k_{y}$, i.e., the energy of spin-up (spin-down) electron decreases (increases) with increasing in-plane momentum $k_{y}$, and become degenerate for higher in-plane momentum $k_{y}$. This phenomenon arises from the difference in interwell coupling between the spin-up and spin-down electrons. The reason is that the height of the middle barrier is varied by changing the magnetic field via the $s-d$ exchange interaction between the electron and the magnetic ions in the DMS layer region. The height of the middle DMS layer is enhanced for the spin-up electron but weakened for the spin-down electron, and the barrier height and the well depth increases with increasing magnetic fields. At small magnetic field [see Fig. 1(a)], the DMS layer is a barrier for both the spin-up and the spindown electron and the interwell coupling leads to a decrease of the electron energy with small in-plane momentum. At high magnetic field [see Fig. 1(b)], the DMS layer is a barrier for the spin-up electron but becomes a well for the spindown electron. This difference results in a different energy dispersion at small in-plane momentum. The flat energy dispersion for spin-down electrons with small momentum corresponds to the Laudau levels with energy $E=(n$ $+1 / 2) \hbar \omega_{c}, n=0,1,2 \cdots$ [see Fig. 1(b)]. The rapid increase of electron energy at large momentum corresponds to the edge states of the electron. In the insets of Figs. 1(a) and 1(b) we plot the group velocity of the electrons as a function of the Fermi energy for the three lowest subbands. The velocity of the electron for the different spin orientations are very different for low energy (small $k_{y}$ ), but become the same for high energy (large $k_{y}$ ).
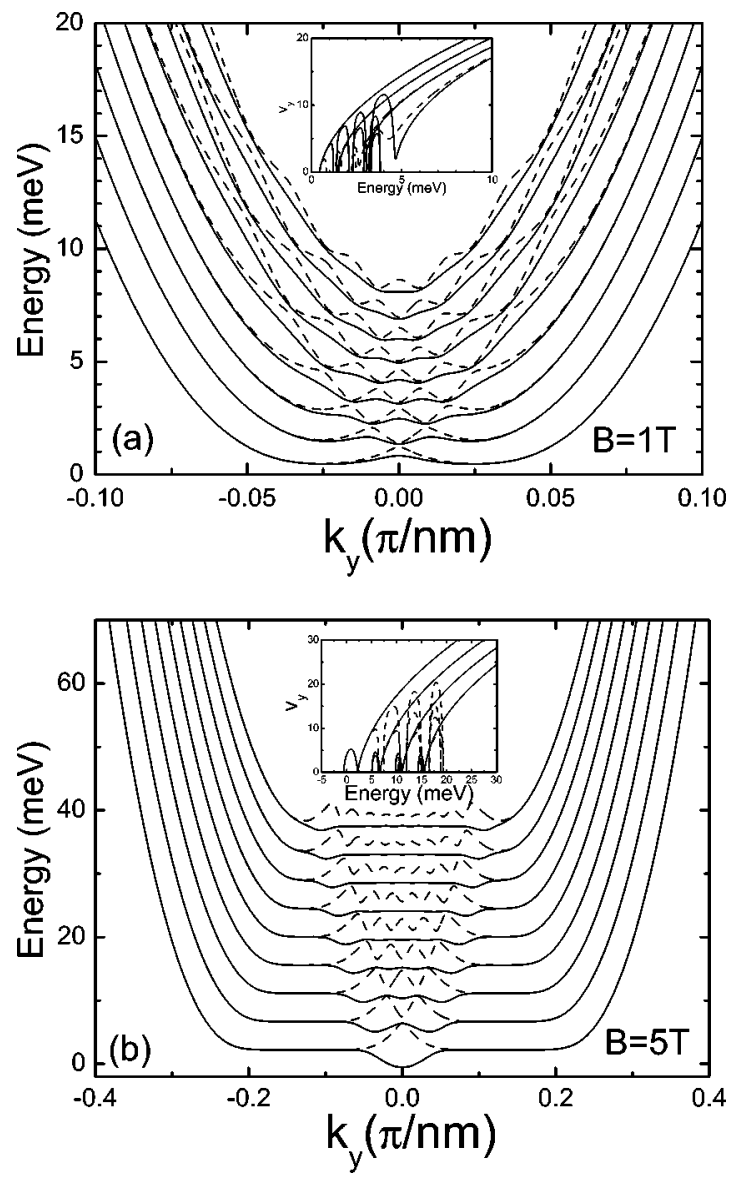

FIG. 1. The energy dispersion of an electron in a DMS DQW for (a) $B=1 \mathrm{~T}$ and (b) $B=5 \mathrm{~T}$. The well width is $W=100 \mathrm{~nm}$ and the thickness of the middle barrier is $d=10 \mathrm{~nm}$. The in-plane momentum $k_{y}$ is in unit of $\pi / \mathrm{nm}$. The solid (dashed) curves depict the energy dispersion for spin-down (spin-up) electrons. The inset shows the group velocity of the electron as a function of energy, the dashe (solid) lines denote the group velocity of the spin-up (spindown) electron.

In order to understand the spectrum of the electron for the different spin orientation, we plot the effective potential as a function of the cyclotron center $x_{0}$, i.e., the momentum $p_{y}$ of the electron. From Fig. 2(a) one sees that the effective potential for the spin-up and spin-down electron are quite different, i.e., the DMS layer is barrier for the spin-up electron, but a well for the spin-down electron. This difference is caused by the $s-d$ exchange interaction between the electron and the magnetic ions, and results in the different behavior of the energy dispersion of the electron with different spin orientation. The interwell coupling for the spin-up electron leads to a decrease of the energy, and the enhancement of the confinement increases the energy of the spin-down electron [see Fig. 2(b)]. When the electron momentum increases, i.e., the cyclotron center shifts towards the side of DQW, this difference of the effective potential is weakened and disappear when the cyclotron center is far away from the DMS layer [see Figs. 2(c) and 2(d)]. Therefore the energies of the spin-up and spin-down electron become degenerate.

The probability distribution for the electron in the DMS DQW is shown in Figs. 3(a) and 3(b) for different in-plane 

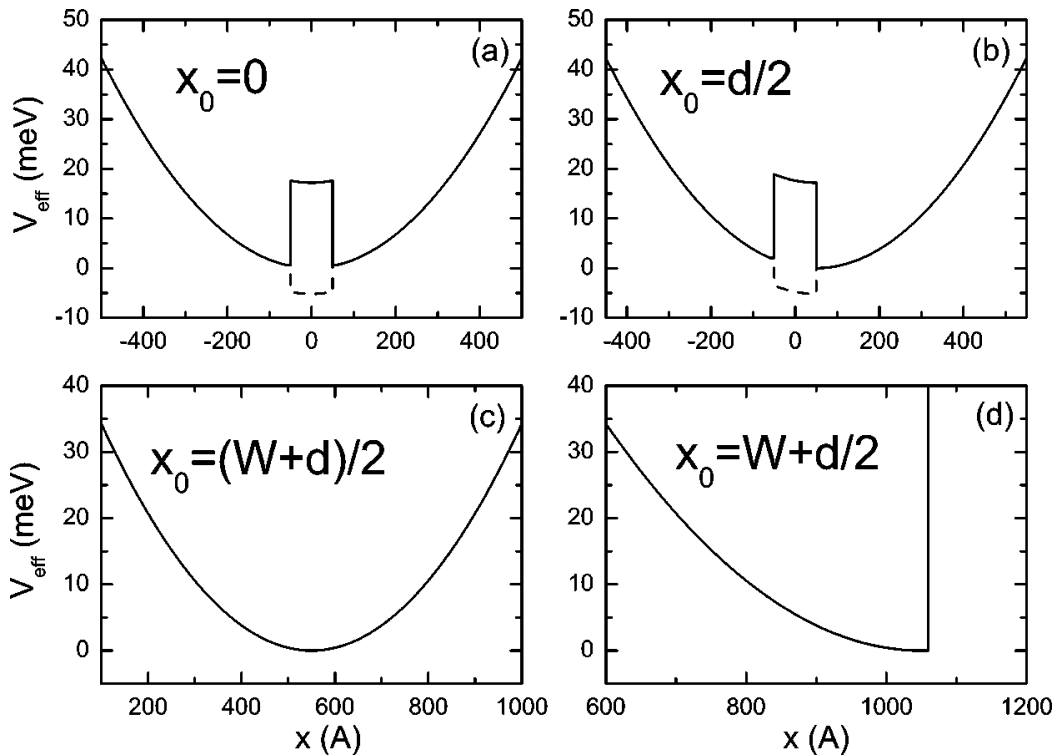

FIG. 2. The effective potential of the electron states in a DMS DQW for different cyclotron centers $x_{0}=-p_{y} / e B$, i.e., different in-plane momentum $p_{y}$. The structural parameters are the same as in Fig. 1. momentum. The inset in Fig. 3(a) shows schematically the band profile for $k_{y}=0$ of DMS DQW structure. At small magnetic field, the spin-up and the spin-down electrons are both located at the two sides of the DMS layer when the
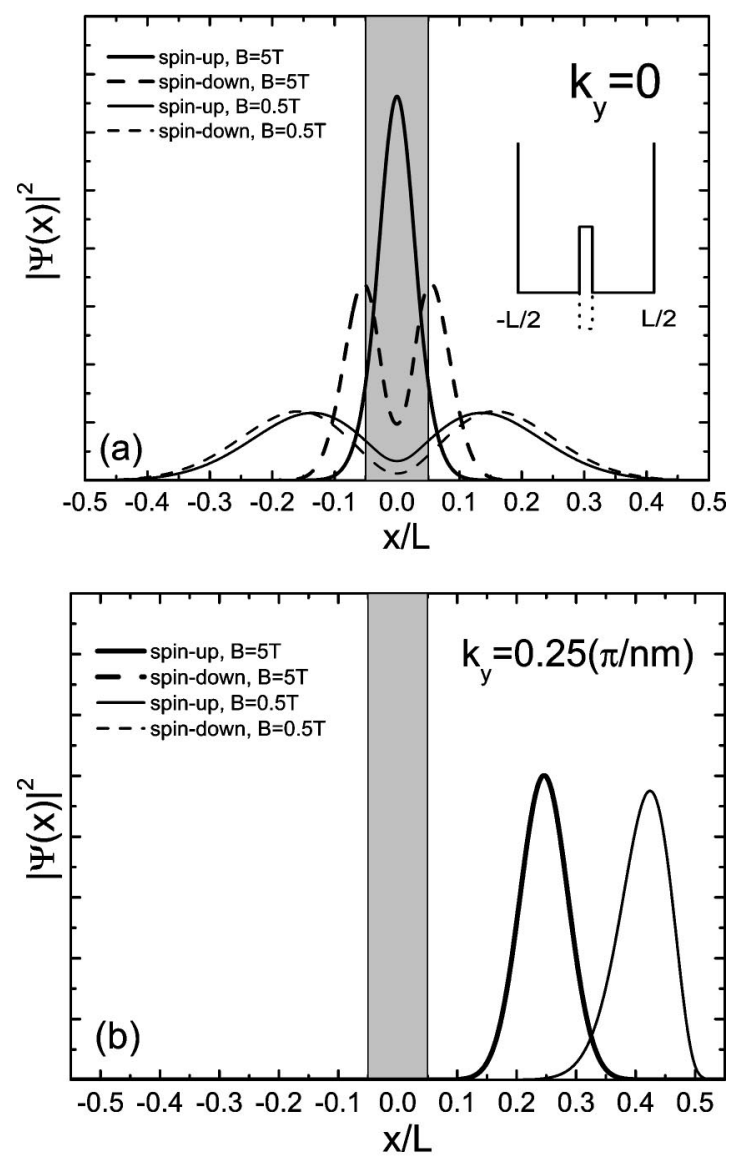

FIG. 3. The probabilities of the electron states in the lowest subband in a DMS DQW for different in-plane momentum and magnetic fields. The structural parameters are the same as in Fig. 1. The shaded region denotes the DMS layer. in-plane momentum $k_{y}=0$ [see Fig. 3(a)], the probabilities are slightly different due to the different barrier heights. But for higher magnetic field, the DMS layer is still a barrier for the spin-up electrons but becomes a well for the spin-down electron, therefore the spin-down electron localizes at the center of the DMS layer, but the spin-up electron is still distributed at the two sides of the DMS layer. For finite inplane momentum $k_{y}$, the Lorentz force pushes the spin-up and spin-down electron towards one side of the DQW where the local confining potentials felt by spin-up and spin-down electron are almost the same [see Figs. 2(c) and 2(d)] resulting in very similar probability distributions for spin-up and spin-down electron states with equal energy [see Fig. 1].

Figures 4(a) and 4(b) show the conductance of the electrons as a function of the Fermi energy for two different magnetic fields. Many interesting spin-dependent peaks and dips can be observed in this figure. When the magnetic field is small, the conductance of the electron exhibits a steplike characteristic as a function of the Fermi energy. The conductance exhibits many peaks and dips with increasing Fermi energy for higher magnetic fields. These structures are caused by the variation of the electron energy dispersion is tuned by the magnetic field and the $s-d$ exchange interaction. At small $k_{y}$ the electron energy oscillates with increasing in-plane momentum $k_{y}$. In the insets of Figs. 4(a) and 4(b) we show the conductance as a function of the magnetic fields. The conductance exhibits a local minimum with increasing magnetic field. In the insets, the spin polarization (SP) of the conductance in the DQW is plotted as a function of the Fermi energy. The SP oscillates with increasing Fermi energy in the presence of magnetic field. This is caused by the opening and quenching of the electron channel in the DQW for different spin orientation with increasing Fermi energy.

Figures 5(a) and 5(b) depict the conductance of the electron as a function of magnetic field for different electron densities. The conductance exhibits an oscillating behavior and decreases with increasing magnetic fields. From the inset 

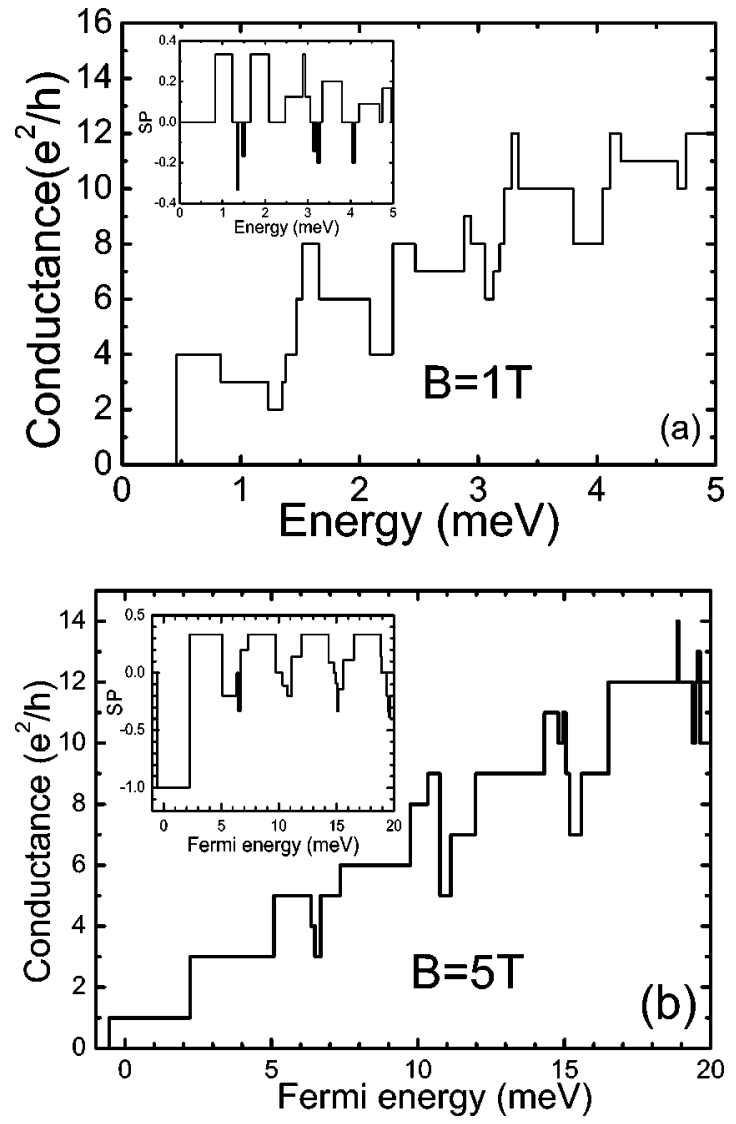

FIG. 4. The conductance vs the Fermi energy in a DMS DQW for two different magnetic fields: (a) $B=1 \mathrm{~T}$ and (b) $B=5 \mathrm{~T}$. The inset shows the spin polarization of the conductance.

one sees that the SP oscillates and increases when the magnetic field increases. Since the conductance is proportional to the number of channels below the Fermi energy [see Eq. (5)], and the energy of the different subbands increases with increasing magnetic field, a conduction channel is closed when the bottom of the subband sweeps across the Fermi surface. The energy difference between the spin-up and spin-down electron subband leads to oscillations in the spin polarization. Figure 6 plots the conductance of the electron versus magnetic fields for the different structures, i.e., the width of well and the thickness of DMS layer. Because the barrier height of the DMS layer can be tunned by changing magnetic fields, the energy dispersion of the electron in DQW exhibits the oscillatory behavior at small momentum $k_{y}$ [see Figs. 1(a) and 1(b)]. The oscillation of conductance can also be observed since this oscillation arises from the variation of the channel number at the Fermi energy. The Fermi energy sweeps across the local maximums and minimums of energy dispersion i.e., quenching or opening of the channel, when the magnetic field changes. Therefore the interwell coupling and Fermi energy play important role on the oscillation of conductance. From our numerical results, the higher Fermi energy and the low magnetic fields $(B<3 \mathrm{~T})$ could lead to the stronger oscillation of the electron conductance.

In conclusion, we investigated the magnetotransport in DMS DQW and found a spin-dependent structure with in-
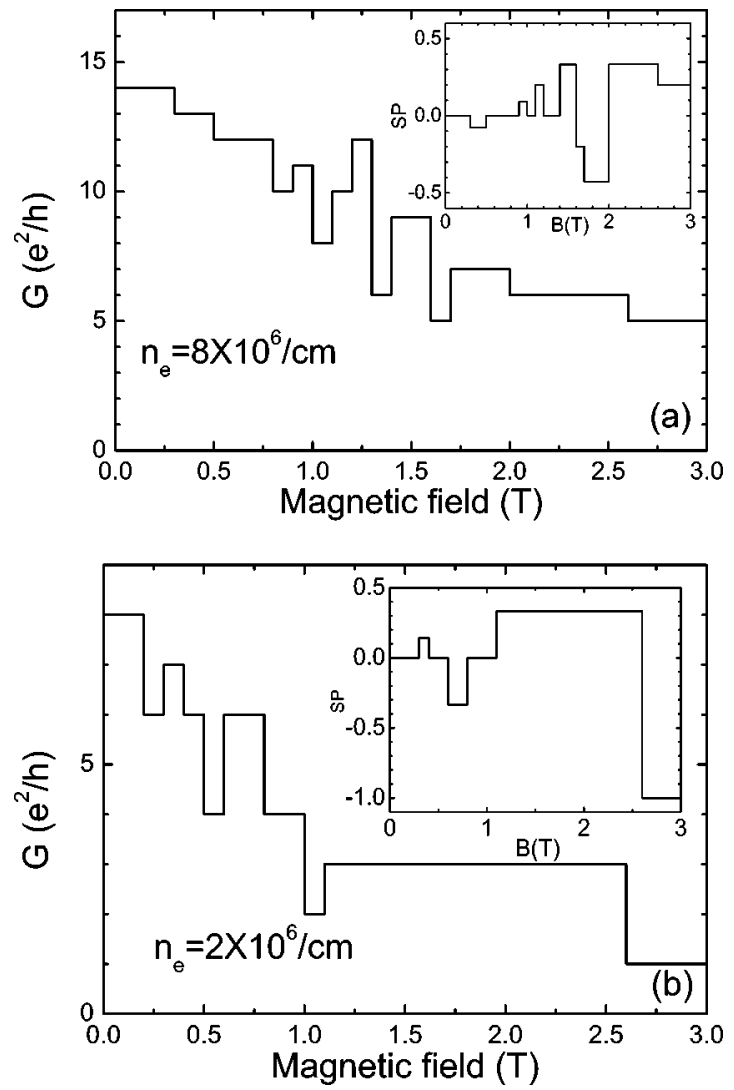

FIG. 5. The conductance of electron vs magnetic fields in a DMS DQW for two different electron densities. The inset shows the spin polarization of the conductance as a function of magnetic field.

creasing Fermi energy and magnetic field. The spin polarization oscillates as function of the Fermi energy in the presence of an external magnetic field. This type of spin-dependent conductance is caused by the spin-dependent interwell coupling which can be tuned by changing the external magnetic field via the $s-d$ exchange interaction. In the DMS DQW system, magnetic fields can modify the electron energy dis-

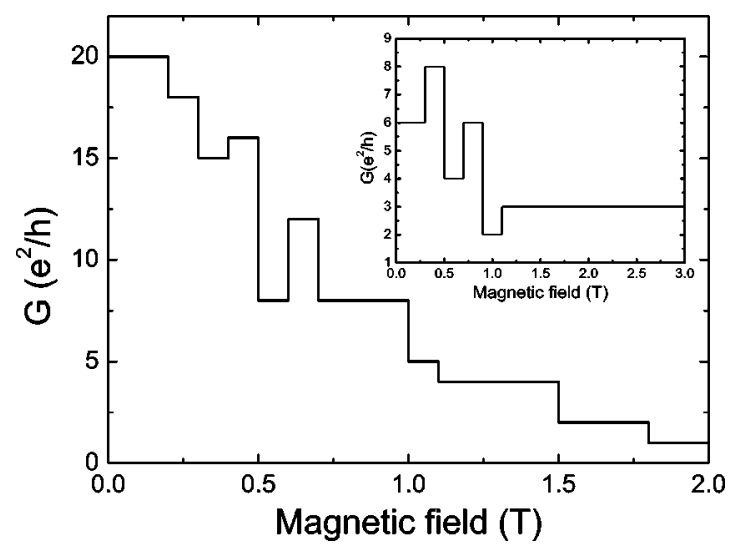

FIG. 6. The conductance of electron vs magnetic fields in a DMS DQW $D_{b}=10 \mathrm{~nm}, W=200 \mathrm{~nm}, n_{e}=8 \times 10^{6} / \mathrm{cm}$. The inset shows the conductance as a function of magnetic field for different structure and electron density. $D_{b}=5 \mathrm{~nm}, W=100 \mathrm{~nm}, n_{e}=2$ $\times 10^{6} / \mathrm{cm}$. 
persion significantly as a function of the in-plane momentum and consequently influences the transport property of the electron in the DMS DQW. This spin-polarized transport property is extremely attractive from the point of view of both basic research and potential spintronics device.
This work is partly supported by the NSFC, the special fund for Major State Basic Research Project No. G001CB3095 of China, the Flemish Science Foundation (FWO-Vl), and the Bilateral Cooperation program between Flanders and China.
*Electronic address: kchang@red.semi.ac.cn

${ }^{1}$ G.A. Prinz, Phys. Today 48, 58 (1995); Science 282, 1660 (1998), and references therein.

${ }^{2}$ R. Fiederling, M. Keim, G. Reuscher, W. Ossau, G. Schmidt, A. Waag, and L.W. Molenkamp, Nature (London) 402, 787 (1999); Y. Ohno, D.K. Young, B. Benschoten, F. Matsukura, H. Ohno, and D.D. Awschalom, ibid. 402, 790 (1999).

${ }^{3}$ S. Datta and B. Das, Appl. Phys. Lett. 56, 665 (1990).

${ }^{4}$ C.M. Hu, Junsaku Nitta, A. Jensen, J.B. Hansen, and H. Takayanagi, Phys. Rev. B 63, 125333 (2001).

${ }^{5}$ B.J. van Wees, H. van Houten, C.W.J. Beenakker, J.G. William- son, L.P. Kouwenhoven, D. van der Marel, and C.T. Foxon, Phys. Rev. Lett. 60, 848 (1988).

${ }^{6}$ Physics of II-VI and I-VII Compounds, Semimagnetic Semiconductors, edited by O. Madelung, M. Schulz, and H. Weiss (Springer, Berlin, 1982), Vol. 17.

${ }^{7}$ S. Lee, M. Dobrowolska, J.K. Furdyna, H. Luo, and L.R. RamMahan, Phys. Rev. B 54, 16939 (1996).

${ }^{8}$ H. Jeon, J. Ding, W. Patterson, A.V. Nurmikko, W. Xie, D.C. Grillo, M. Kobayashi, and R.L. Gunshor, Appl. Phys. Lett. 59, 3619 (1991).

${ }^{9}$ M. Büttiker, Phys. Rev. B 38, 9375 (1988). 